\title{
The Turbulent Chiral Magnetic Cascade in the Early Universe
}

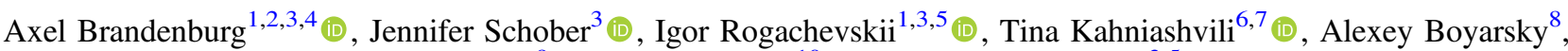 \\ Jürg Fröhlich ${ }^{9}$, Oleg Ruchayskiy ${ }^{10}{ }^{(D)}$, and Nathan Kleeorin ${ }^{3,5}$ (D) \\ ${ }^{1}$ Laboratory for Atmospheric and Space Physics, University of Colorado, 3665 Discovery Drive, Boulder, CO 80303, USA \\ ${ }^{2}$ JILA and Department of Astrophysical and Planetary Sciences, University of Colorado, Box 440, Boulder, CO 80303, USA \\ ${ }^{3}$ Nordita, KTH Royal Institute of Technology and Stockholm University, Roslagstullsbacken 23, SE-10691 Stockholm, Sweden \\ ${ }^{4}$ Department of Astronomy, AlbaNova University Center, Stockholm University, SE-10691 Stockholm, Sweden \\ ${ }^{5}$ Department of Mechanical Engineering, Ben-Gurion University of the Negev, P.O. Box 653, Beer-Sheva 84105, Israel \\ ${ }^{6}$ McWilliams Center for Cosmology and Department of Physics, Carnegie Mellon University, 5000 Forbes Avenue, Pittsburgh, PA 15213, USA \\ ${ }^{7}$ Abastumani Astrophysical Observatory, Ilia State University, 3-5 Cholokashvili Street, 0194 Tbilisi, Georgia \\ ${ }^{8}$ Instituut-Lorentz for Theoretical Physics, Universiteit Leiden, Niels Bohrweg 2, 2333 CA Leiden, The Netherlands \\ ${ }^{9}$ Institute of Theoretical Physics, ETH Hönggerberg, CH-8093 Zurich, Switzerland \\ ${ }^{10}$ Discovery Center, Niels Bohr Institute, Blegdamsvej 17, DK-2100 Copenhagen, Denmark \\ Received 2017 July 11; revised 2017 August 3; accepted 2017 August 8; published 2017 August 22
}

\begin{abstract}
The presence of asymmetry between fermions of opposite handedness in plasmas of relativistic particles can lead to exponential growth of a helical magnetic field via a small-scale chiral dynamo instability known as the chiral magnetic effect. Here, we show, using dimensional arguments and numerical simulations, that this process produces through the Lorentz force chiral magnetically driven turbulence. A $k^{-2}$ magnetic energy spectrum emerges via inverse transfer over a certain range of wavenumbers $k$. The total chirality (magnetic helicity plus normalized chiral chemical potential) is conserved in this system. Therefore, as the helical magnetic field grows, most of the total chirality gets transferred into magnetic helicity until the chiral magnetic effect terminates. Quantitative results for height, slope, and extent of the spectrum are obtained. Consequences of this effect for cosmic magnetic fields are discussed.
\end{abstract}

Key words: dynamo - early universe - magnetic fields - magnetohydrodynamics (MHD) - turbulence

\section{Introduction}

Asymmetry between the number densities of left- and righthanded fermions gives rise to what is known as the chiral magnetic effect (CME) — an electric current flowing along the magnetic field. This quantum effect was first found by Vilenkin (1980) and then rederived using different arguments (Redlich \& Wijewardhana 1985; Alekseev et al. 1998; Fröhlich \& Pedrini 2000; Fukushima et al. 2008). Joyce \& Shaposhnikov (1997) and Fröhlich \& Pedrini (2000) showed that this phenomenon destabilizes a weak magnetic field and leads to its exponential growth. The CME has applications in many fields of physics ranging from the early universe to neutron stars and condensed matter systems (for reviews, see, e.g., Kharzeev 2014; Miransky \& Shovkovy 2015).

The total chirality in the system, i.e., the sum of magnetic helicity and fermion chiral asymmetry, is conserved. As the field becomes fully helical, the chiral asymmetry will eventually disappear, so the total growth of magnetic fields is limited (Joyce \& Shaposhnikov 1997; Fröhlich \& Pedrini 2000; Tashiro et al. 2012; Giovannini 2013; Boyarsky et al. 2015; Hirono et al. 2015; Pavlović et al. 2017).

There is now significant interest in the possibility of generating a turbulent inverse cascade by the CME (Boyarsky et al. 2012, 2015; Hirono et al. 2015; Dvornikov \& Semikoz 2017; Pavlović et al. 2017). Meanwhile, there has been considerable progress in our understanding of magnetically dominated helical turbulence (Biskamp \& Müller 1999; Kahniashvili et al. 2013). In particular, the magnetic field $\boldsymbol{B}$ decays with time $t$ such that $\left\langle\boldsymbol{B}^{2}\right\rangle \propto t^{-2 / 3}$ while the correlation length grows like $\xi_{\mathrm{M}} \propto t^{2 / 3}$, with $\left\langle\boldsymbol{B}^{2}\right\rangle \xi_{\mathrm{M}}=$ const. In this Letter, we assess the importance of the CME in establishing initial conditions for the turbulent decay. This provides a critical starting point because we predict the value of $\left\langle\boldsymbol{B}^{2}\right\rangle \xi_{\mathrm{M}}$ based on the initial asymmetry.

It is worth noting that observational limits on the product $\left\langle\boldsymbol{B}^{2}\right\rangle \xi_{\mathrm{M}}$ have been derived from the non-observations of $\mathrm{GeV}$ energy halos around TeV blazars (Aharonian et al. 2006). This has been interpreted in terms of magnetic fields permeating the intergalactic medium over large scales (for a review, see Durrer $\&$ Neronov 2013). Simultaneous GeV-TeV observations of blazars put lower limits on such fields between $10^{-15} \mathrm{G}$ (Taylor et al. 2011) and $10^{-18} \mathrm{G}$ (Dermer et al. 2011) at $\xi_{\mathrm{M}} \sim 1 \mathrm{Mpc}$. Systematic parity-odd correlations between the directions of secondary photons and their energies from the surroundings of blazars have been interpreted in terms of helical magnetic fields of the order of $10^{-14} \mathrm{G}$ (Tashiro et al. 2014; Tashiro \& Vachaspati 2015). If this can be independently confirmed, it would be a real detection.

The present-day value of $\left\langle\boldsymbol{B}^{2}\right\rangle \xi_{\mathrm{M}}$ and, more generally, the modulus of the magnetic helicity can be constrained on dimensional grounds under the assumption that it is determined only by the present-day temperature $T_{0}$ plus fundamental constants: the Boltzmann constant $k_{\mathrm{B}}$, the reduced Planck constant $\hbar$, and the speed of light $c$. As the dimension of $\left\langle\boldsymbol{B}^{2}\right\rangle$ is erg $\mathrm{cm}^{-3}=\mathrm{G}^{2} / 4 \pi$, we find

$$
\left\langle\boldsymbol{B}^{2}\right\rangle \xi_{\mathrm{M}}=\epsilon\left(k_{\mathrm{B}} T_{0}\right)^{3}(\hbar c)^{-2},
$$

where $\epsilon$ is a dimensionless number (we determine a more precise value in Section 4). Assuming for now $\epsilon=1$ and using $T_{0}=2.75 \mathrm{~K}$, Equation (1) yields the numerical value $\sim 5 \times$ $10^{-14} \mathrm{erg} \mathrm{cm}{ }^{-2}=2 \times 10^{-37} \mathrm{G}^{2} \mathrm{Mpc}$, corresponding to $0.5 \times$ $10^{-18} \mathrm{G}$ at $1 \mathrm{Mpc}$, and thus to the estimate of Dermer et al. (2011). Conversely, if this argument turned out to fail, it might suggest that other dimensionful quantities such as Newton's 
constant might enter. This argument is very rough, and so quantitative models are needed to determine $\epsilon$.

The purpose of this Letter is to examine the onset of turbulence by the chiral small-scale dynamo to compute the spectrum, scale separation, and the saturation level of the resulting magnetic field. We use three-dimensional simulations to verify scaling relations. We conclude with a discussion of the parameters relevant to the early universe and, in particular, the strength of the observable magnetic field.

\section{Growth and Saturation}

The CME can lead to exponential growth owing to a term in the induction equation that is formally similar to the $\alpha$ effect in mean-field dynamo theory (Moffatt 1978; Krause \& Rädler 1980). The important difference is of course that in chiral magnetohydrodynamics we are concerned with the actual magnetic field rather than its macroscopic average. However, much of the intuition from mean-field electrodynamics carries over to chiral magnetohydrodynamics. The mathematical formalism and the underlying fully nonlinear spatially dependent evolution equations were derived by Boyarsky et al. (2015) and analyzed by Rogachevskii et al. (2017).

Since $\boldsymbol{B}$ is solenoidal, we express it as $\boldsymbol{B}=\boldsymbol{\nabla} \times \boldsymbol{A}$. We define $\mu=24 \alpha_{\mathrm{em}}\left(n_{\mathrm{L}}-n_{\mathrm{R}}\right)\left(\hbar c / k_{\mathrm{B}} T\right)^{2}$ as the normalized chemical potential, where $\alpha_{\mathrm{em}} \approx 1 / 137$ is the fine structure constant, and $n_{\mathrm{L}}$ and $n_{\mathrm{R}}$ are the number densities of left- and right-handed fermions, respectively. The governing equations for $\boldsymbol{A}$ and $\mu$ are

$$
\begin{gathered}
\frac{\partial \boldsymbol{A}}{\partial t}=\eta(\mu \boldsymbol{B}-\nabla \times \boldsymbol{B})+\boldsymbol{U} \times \boldsymbol{B}, \\
\frac{D \mu}{D t}=-\lambda \eta(\mu \boldsymbol{B}-\nabla \times \boldsymbol{B}) \cdot \boldsymbol{B}+D \nabla^{2} \mu-\Gamma_{\mathrm{f}} \mu,
\end{gathered}
$$

where $D / D t \equiv \partial / \partial t+\boldsymbol{U} \cdot \boldsymbol{\nabla}$ is the advective derivative, $\eta$ is the magnetic diffusivity (not to be confused with the conformal time $\left.^{11}\right), \lambda \equiv 3 \hbar c\left(8 \alpha_{\mathrm{em}} / k_{\mathrm{B}} T\right)^{2}$ characterizes the feedback of the electromagnetic field on the evolution of $\mu, D$ is a chiral diffusion coefficient, $\Gamma_{\mathrm{f}}$ is the flipping rate, and $\boldsymbol{U}$ is the plasma velocity, which obeys the usual momentum equation and the continuity equation for the density $\rho$,

$$
\begin{gathered}
\rho \frac{D \boldsymbol{U}}{D t}=(\boldsymbol{\nabla} \times \boldsymbol{B}) \times \boldsymbol{B}-\nabla p+\nabla \cdot(2 \rho \nu \mathbf{S}), \\
\frac{D \rho}{D t}=-\rho \boldsymbol{\nabla} \cdot \boldsymbol{U},
\end{gathered}
$$

where $\mathrm{S}_{i j}=1 / 2\left(U_{i, j}+U_{j, i}\right)-\frac{1}{3} \delta_{i j} \nabla \cdot \boldsymbol{U}$ is the rate-of-strain tensor, $\nu$ is the viscosity, and $p$ is the pressure, which is assumed to be proportional to the density, i.e., $p=\rho c_{\mathrm{s}}^{2}$, with $c_{\mathrm{s}}$ being the speed of sound; for a gas of ultra-relativistic particles, $c_{\mathrm{s}}^{2}=c^{2} / 3$.

The magnetic field is normalized such that the magnetic energy density is $\boldsymbol{B}^{2} / 2$ without the $4 \pi$ factor. The usual magnetic field in Gauss is therefore $\sqrt{4 \pi} \boldsymbol{B}$. Furthermore, if $\mu=\lambda=0$, we recover the usual hydromagnetic equations. The physical values of $\lambda$ and $\eta$, as well as the initial values

\footnotetext{
11 Our evolution equations are also valid in an expanding universe when interpreting $t$ as conformal time and using comoving quantities; see Brandenburg et al. (1996), whose equations contain extra terms and 4/3 factors that affect $\rho$ and $\boldsymbol{u}$ only slightly and do not affect our results.
}

of $\mu$, will be discussed in Section 4 in the context of the early universe. In the following, however, we consider a broad parameter space and are particularly interested in the case where the magnetic field can grow out of a weak seed due to the CME.

An important consequence of Equations (2) and (3) is the conservation of (volume-averaged) total chirality:

$$
1 / 2 \lambda\langle\boldsymbol{A} \cdot \boldsymbol{B}\rangle+\langle\mu\rangle=\mathrm{const} \equiv \mu_{0} \quad\left(\text { for } \Gamma_{\mathrm{f}} \ll \eta \mu_{0}^{2}\right),
$$

where $\mu_{0}$ denotes the initial total chirality and the brackets denote averaging over a closed or periodic volume. ${ }^{12}$ Equation (6) imposes an important constraint on the coevolution of $\mu$ and $\boldsymbol{B}$, and implies

$$
\left\langle\boldsymbol{B}^{2}\right\rangle \xi_{\mathrm{M}} \lesssim \mu_{0} / \lambda
$$

where $\xi_{\mathrm{M}}$ is the correlation length discussed in Section 1.

The initial growth can be described by the linearized equations. Assuming $\boldsymbol{B}(t, \boldsymbol{x}) \propto \exp (\gamma t+\mathrm{i} \boldsymbol{k} \cdot \boldsymbol{x})$, where $\gamma$ is the dynamo growth rate and $\boldsymbol{k}$ is the wavevector, the dispersion relation becomes $\gamma(k)=\eta k(\mu-k)$, where $k=|\boldsymbol{k}|$ is the wavenumber. This dispersion relation predicts magnetic field growth for $k<\mu$. The linear approximation is applicable as long as the field is weak and nonlinear effects are small. In a domain of size $L$, which could represent the horizon scale in the early universe or the length of a periodic computational domain, there will be a minimum wavenumber $k_{1}=2 \pi / L$. A weak seed magnetic field is only unstable if $k_{1}<\mu$. In addition, there is a wavenumber $k_{\max }$ at which the growth rate is maximum, i.e., $d \gamma / d k=0$. It is given by $k_{\max }=\mu / 2$. Eventually, following the early exponential growth, the magnetic field reaches a critical value and a more complicated nonlinear stage commences.

The CME introduces two new quantities into the system: $\lambda$ and $\mu$. Different evolutionary scenarios can be envisaged depending on their values. For the purpose of this discussion, we ignore the fact that $\mu$ is changing as $\boldsymbol{B}$ evolves; all normalizations below are actually based on $\mu_{0}$. Using the fact that $\lambda^{-1}$ has the dimension of energy per unit length and $\mu$ has the dimension of inverse length, we can identify two characteristic velocities:

$$
v_{\lambda}=\mu /(\bar{\rho} \lambda)^{1 / 2}, \quad v_{\mu}=\mu \eta,
$$

where $\bar{\rho}$ is the mean density of the plasma. Assuming that both velocities are well below the speed of sound, $c_{\mathrm{s}}$, we can identify two regimes of interest:

$$
\begin{gathered}
c_{\mathrm{S}}>v_{\lambda}>v_{\mu}>\eta k_{1} \quad(\text { regime } \mathrm{I}), \\
c_{\mathrm{S}}>v_{\mu}>v_{\lambda}>\eta k_{1} \quad(\text { regime } \mathrm{II}) .
\end{gathered}
$$

In regime $\mathrm{I}$, the ratio $v_{\lambda} / v_{\mu}=\left[\eta(\bar{\rho} \lambda)^{1 / 2}\right]^{-1}$ is large, so the $\lambda$ term is unimportant and $\mu$ will only change slowly as the magnetic field grows. Once the magnetic field exceeds a critical value of around $\sqrt{\bar{\rho}} \mu \eta$, i.e., when the magnetic Reynolds number exceeds a certain value, the magnetic field becomes turbulent. However, the field continues to grow until the Alfvén speed $v_{\mathrm{A}}=B / \sqrt{\bar{\rho}}$ approaches $v_{\lambda}$. At that point, $\mu$ begins to be depleted, as is clear from Equation (6). This quenches further growth, and magnetic energy can then only decay. In regime II,

\footnotetext{
12 At high temperatures we neglect the influence of the fermions' mass that would gradually destroy the total chirality (6).
} 
the ratio $v_{\lambda} / v_{\mu}$ is small, so the $\lambda$ term is important and $\mu$ will be depleted before a turbulent cascade develops. As in regime I, the magnetic energy must eventually decay. In both cases, however, since the magnetic field is maximally helical, the decay will be slower than for a nonhelical field and will be accompanied by a strong, self-similar inverse transfer (Christensson et al. 2001; Brandenburg \& Kahniashvili 2017).

In the following, we will see that a more accurate distinction between regimes I and II occurs when $v_{\lambda} / v_{\mu} \approx 8$ instead of unity. For this purpose, let us discuss the resulting magnetic spectrum in more detail. The spectrum is defined such that $\int E_{\mathrm{M}}(k, t) d k=\left\langle\boldsymbol{B}^{2}\right\rangle / 2$. Thus, $E_{\mathrm{M}}(k, t)$ has the dimension of $\bar{\rho} \mu \eta^{2}$, so it will be convenient to normalize $E_{\mathrm{M}}(k, t)$ correspondingly. In magnetically dominated turbulence, where the velocity is just a consequence of driving by the Lorentz force, we expect weak turbulence scaling (Galtier et al. 2000) with a spectrum proportional to $k^{-2}$. This was originally thought to be applicable to the case with an external magnetic field, but this spectrum was found also for the isotropic case of magnetically dominated turbulence (Brandenburg et al. 2015). On dimensional grounds, we can expect a spectrum with an inertial range of the form

$$
E_{\mathrm{M}}(k, t)=C_{\mu} \bar{\rho} \mu^{3} \eta^{2} k^{-2},
$$

where $C_{\mu}$ is a chiral magnetic Kolmogorov-type constant. Note that $\lambda$ does not enter in Equation (11). This cannot be justified by dimensional arguments alone and requires verification from simulations that will be presented below. It should be noted, however, that $\lambda$ characterizes the ultimate depletion of $\mu$ after turbulence develops and the magnetic field saturates.

The depletion of $\mu$, and therefore of the CME, becomes stronger with increasing values of $\lambda$, thus limiting the magnetic field to progressively smaller values as $\lambda$ is increased. Using Equation (7), we find that

$$
E_{\mathrm{M}}(k, t) \leqslant C_{\lambda} \mu / \lambda,
$$

where $C_{\lambda}$ is another Kolmogorov-type constant describing the saturation caused by $\lambda$. Note that this limit for $E_{\mathrm{M}}(k, t)$ is independent of $k$ and applicable to all $k \leqslant k_{\lambda}$, where $k_{\lambda}$ is a critical value that will be estimated below.

Equation (12) suggests that in regime I, we have an inertial range for $E_{\mathrm{M}}(k, t)<C_{\lambda} \mu / \lambda$ with a $k^{-2}$ spectrum down to the smallest excited scale at wavenumber $k=\mu$. Using Equations (11) and (12), we can determine a critical value $k_{\lambda}$ as

$$
k_{\lambda}=\sqrt{\bar{\rho} \lambda C_{\mu} / C_{\lambda}} \mu \eta \text {. }
$$

The resulting $k^{-2}$ spectrum in $k_{\lambda}<k<\mu$ is sketched in Figure 1. It is now clear that the crossover between regimes I and II occurs when $k_{\lambda} \approx \mu / 2$, i.e., when $v_{\lambda} / v_{\mu} \approx 2 \sqrt{C_{\mu} / C_{\lambda}}$. To determine the constants $C_{\mu}$ and $C_{\lambda}$, we need numerical simulations, which will be presented in the next section.

\section{Simulations}

Below, we present results of three-dimensional simulations in a cubic periodic domain. Detailed analysis of different simulations with chiral magnetically driven turbulence and externally forced turbulence are presented in a separate study (Schober et al. 2017). We use the PENCIL CODE ${ }^{13}$ with $288^{3}$

\footnotetext{
13 https://github.com/pencil-code
}

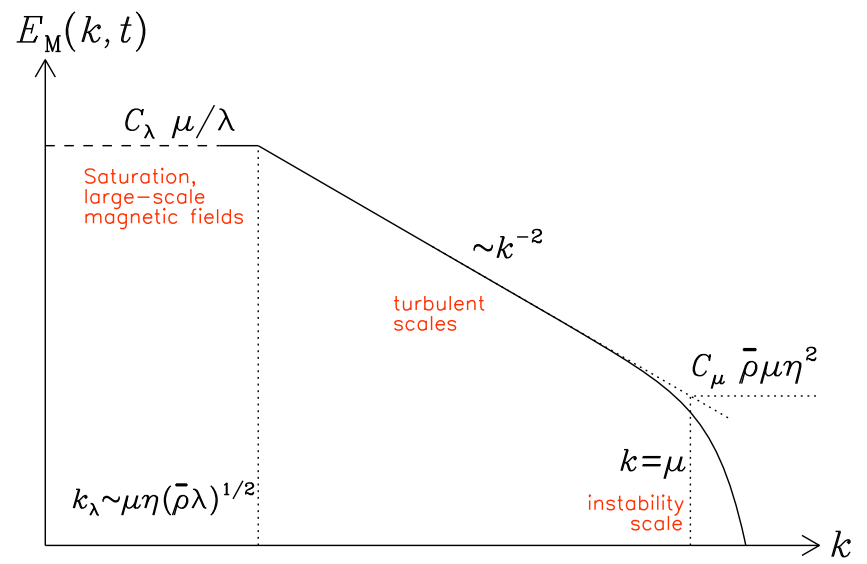

Figure 1. Sketch of the magnetic energy spectrum of chiral magnetically driven turbulence.

and $200^{3}$ mesh points. We vary the values of $\mu$, $\eta$, and $\lambda$, covering both regimes I and II. Numerical stability requires $\nu$ and $D$ to be larger than what is physically realistic, so we take $\nu=D=\eta$ as a compromise. As in earlier work, we assume $\Gamma_{\mathrm{f}}=0$. The governing parameters are listed in Table 1.

In Figure 2, we show magnetic energy spectra for different values of $v_{\lambda} / v_{\mu}$. As explained in Section 2, the depletion of $\mu$ is small if $\lambda$ is small and thus $v_{\lambda}$ is large, so for $v_{\lambda} / v_{\mu} \gg 1$, a turbulent cascade with a power law as in Equation (11) is possible. This is shown in panel (a), where $v_{\lambda} / v_{\mu}=700$, and a $k^{-2}$ spectrum is seen for all $k<\mu$. As $v_{\lambda} / v_{\mu}$ is decreased, depletion of $\mu$ is increased; see Figure 2(b). The limiting line where $E_{\mathrm{M}}(k, t)=\mu / \lambda$ is shown as a dashed-dotted line, and we see that a typical inverse transfer sets in as found previously for decaying turbulence (Christensson et al. 2001; Banerjee \& Jedamzik 2004; Brandenburg \& Kahniashvili 2017).

Next, we discuss the values of $C_{\lambda}$ and $C_{\mu}$. Figures 2(c) and (d) have already demonstrated that $C_{\lambda} \approx 1$ is a good approximation. The value of $C_{\mu}$ can be seen from the intercept of the $k^{-2}$ power law with the $k=\mu$ line in Figures 2(a) and (b). We see that the intercept lies at $C_{\mu} \approx 16$. This implies that the crossover between regimes I and II is at $v_{\lambda} / v_{\mu} \approx 2 \sqrt{C_{\mu}} \approx 8$, which is compatible with Figure 2(c).

For $v_{\lambda} / v_{\mu}<8$, the inverse transfer begins once the approximately monochromatic exponential growth at $k=\mu / 2$ saturates. Both for large and small values of $v_{\lambda} / v_{\mu}$, the magnetic field is turbulent, as shown in Figure 3, where we compare visualizations of $B_{x}$ and $U_{x}$ on the periphery of the computational domain. $\boldsymbol{B}$ attains a large-scale component of Beltrami-type, which is force-free and of the form $\left(\sin k_{1} z, \cos k_{1} z, 0\right)$ with positive helicity. It is a matter of chance in which direction the field varies. Examples of fields varying in any of the other two directions have been found for helically forced turbulent dynamos (Brandenburg 2001).

Initially and at late times, $\mu$ is nearly uniform. At intermediate times, however, the ratio of its rms value to the average can reach $25 \%$ in the case with $v_{\lambda} / v_{\mu}=7$. The typical kinetic energy can reach $12 \%$ of the magnetic energy.

The value of $C_{\mu}$ can be determined more accurately by plotting energy spectra compensated by $k^{2} / \mu^{3} \eta^{2}$; see Figure 4 . We see that $C_{\mu} \approx 16$ is well obeyed for different values of $v_{\mu} / c_{\mathrm{s}}$ and $\mu_{0} / k_{1}$; see Table 1 . Here, we also give the Lundquist number $\mathrm{Lu}=v_{\mathrm{A}} / \eta k_{1}$ and the Reynolds number $\operatorname{Re}=u_{\mathrm{rms}} / \nu k_{1}$ 
Table 1

Summary of the Parameters for the Runs Discussed

\begin{tabular}{|c|c|c|c|c|c|c|c|}
\hline Run & $\mu_{0}$ & $\eta k_{1} / c_{\mathrm{s}}$ & $\bar{\rho} \lambda c_{\mathrm{s}}^{2} / k_{1}^{2}$ & $v_{\mu} / c_{\mathrm{s}}$ & $v_{\lambda} / c_{\mathrm{s}}$ & $\mathrm{Lu}$ & $\operatorname{Re}$ \\
\hline A & 40 & $5 \times 10^{-5}$ & $8 \times 10^{2}$ & 0.002 & 1.4 & $2000^{*}$ & $450^{*}$ \\
\hline B & 40 & $5 \times 10^{-5}$ & $8 \times 10^{4}$ & 0.002 & 0.14 & 830 & 250 \\
\hline $\mathrm{C}$ & 40 & $5 \times 10^{-5}$ & $8 \times 10^{6}$ & 0.002 & 0.014 & 170 & 47 \\
\hline D & 40 & $5 \times 10^{-5}$ & $8 \times 10^{10}$ & 0.002 & $1.4 \times 10^{-4}$ & 1.5 & 0.05 \\
\hline $\mathrm{E}$ & 40 & $5 \times 10^{-5}$ & $10^{2}$ & 0.002 & 4.0 & $2000^{*}$ & 450 \\
\hline $\mathrm{F}$ & 40 & $5 \times 10^{-6}$ & $10^{2}$ & 0.0002 & 4.0 & $2500^{*}$ & $500^{*}$ \\
\hline G & 20 & $5 \times 10^{-6}$ & $10^{2}$ & 0.0001 & 2.0 & $800^{*}$ & 200 \\
\hline
\end{tabular}

Note. $\mu_{0}$ is in units of $k_{1}$. Asterisks denote lower limits.

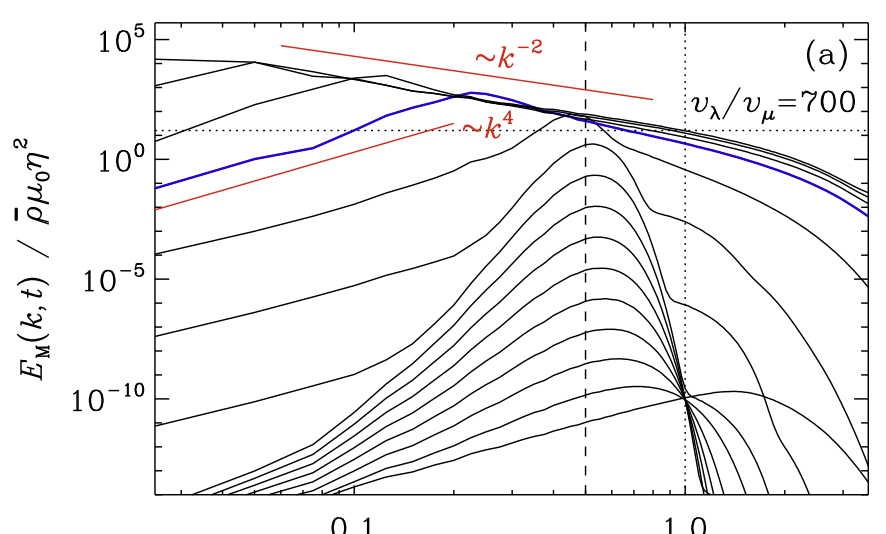

0.1

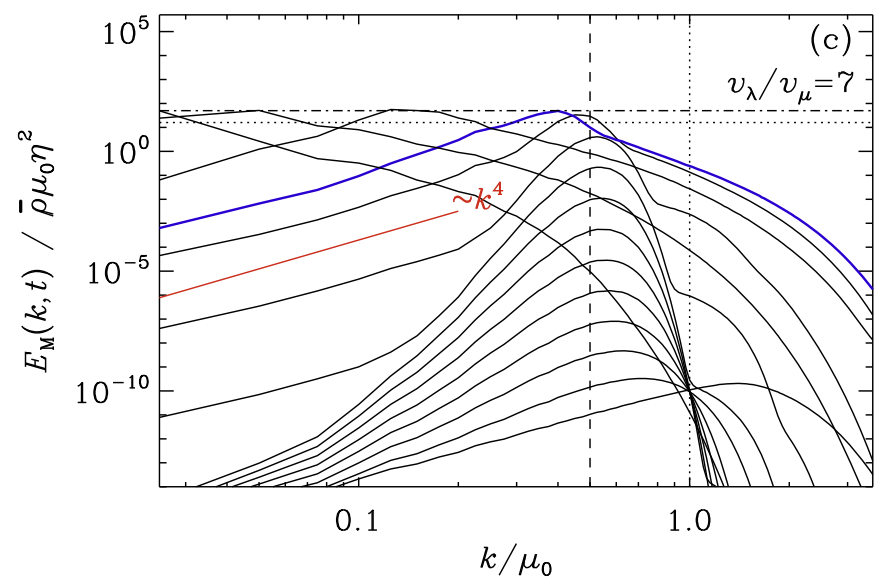

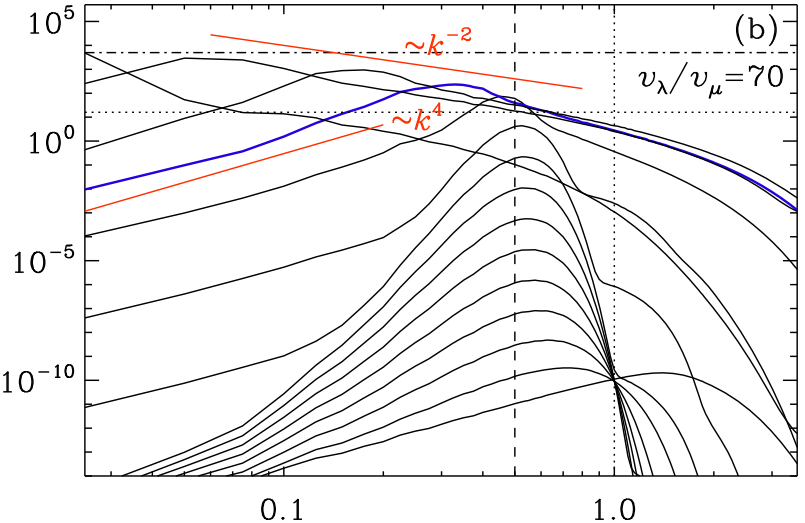

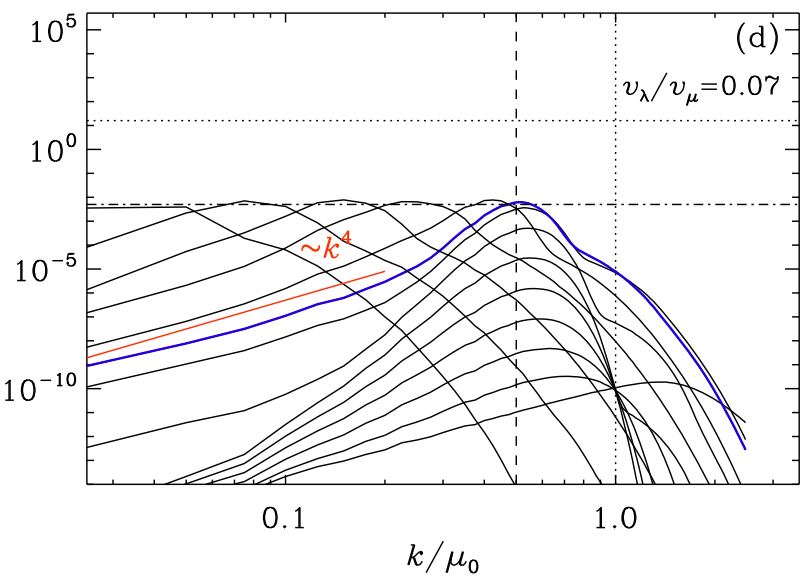

Figure 2. Evolution of magnetic energy spectra for Runs A-D shown in time intervals $\Delta t$ with $\Delta t \eta \mu^{2}=6$ until $t \eta \mu^{2}=80$ (marked in blue) followed by longer time intervals that increase by a factor of two. The vertical dashed line marks the wavenumber $k=\mu_{0} / 2$ where the growth rate is maximum. The horizontal dotted line marks the level of $C_{\mu} \bar{\rho} \mu_{0} \eta^{2}$, and the horizontal dashed-dotted line marks the level of $C_{\lambda} \mu_{0} / \lambda$. The red lines have slopes of -2 and +4 . Panel (d) corresponds to regime II, while panels (a) and (b) correspond to regime I.

at the end of the run. By integrating Equation (11) over $k$, we obtain the estimate

$$
\mathrm{Lu}=\frac{\mu}{k_{1}}\left(\frac{2 C_{\mu} \mu}{k_{\lambda}}\right)^{1 / 2}=\left(C_{\mu} C_{\lambda}\right)^{1 / 4} \frac{\mu}{k_{1}}\left(\frac{2 v_{\lambda}}{v_{\mu}}\right)^{1 / 2} .
$$

This is compatible with the values in Table 1, especially for Run B, while for other runs they are only lower limits.

The early exponential growth of the magnetic field is superficially reminiscent of a turbulent dynamo. A major difference is, however, the absence of an initial transient, which is usually found in dynamo simulations (Brandenburg 2010).
In all cases, we started with a random magnetic field with a Batchelor $k^{4}$ spectrum (Durrer \& Caprini 2003). Looking again at Figures 2(a)-(d), we see that at later times the field depends only on the initial value of $E_{\mathrm{M}}(\mu / 2,0)$ and is independent of the spectral energy at any other $k$, so we have

$$
E_{\mathrm{M}}(\mu / 2, t)=E_{\mathrm{M}}(\mu / 2,0) \exp \left(\gamma_{\max } t\right)
$$

where $\gamma_{\max }=\eta \mu^{2} / 4$ is the value of $\gamma(k)$ at $k=\mu / 2$. At $k=\mu_{0}$, the spectral energy is strictly constant in the kinematic phase, so all lines cross at that one point, where the spectral magnetic energy density is equal to the initial value. The magnetically driven chiral turbulence and resulting large-scale 

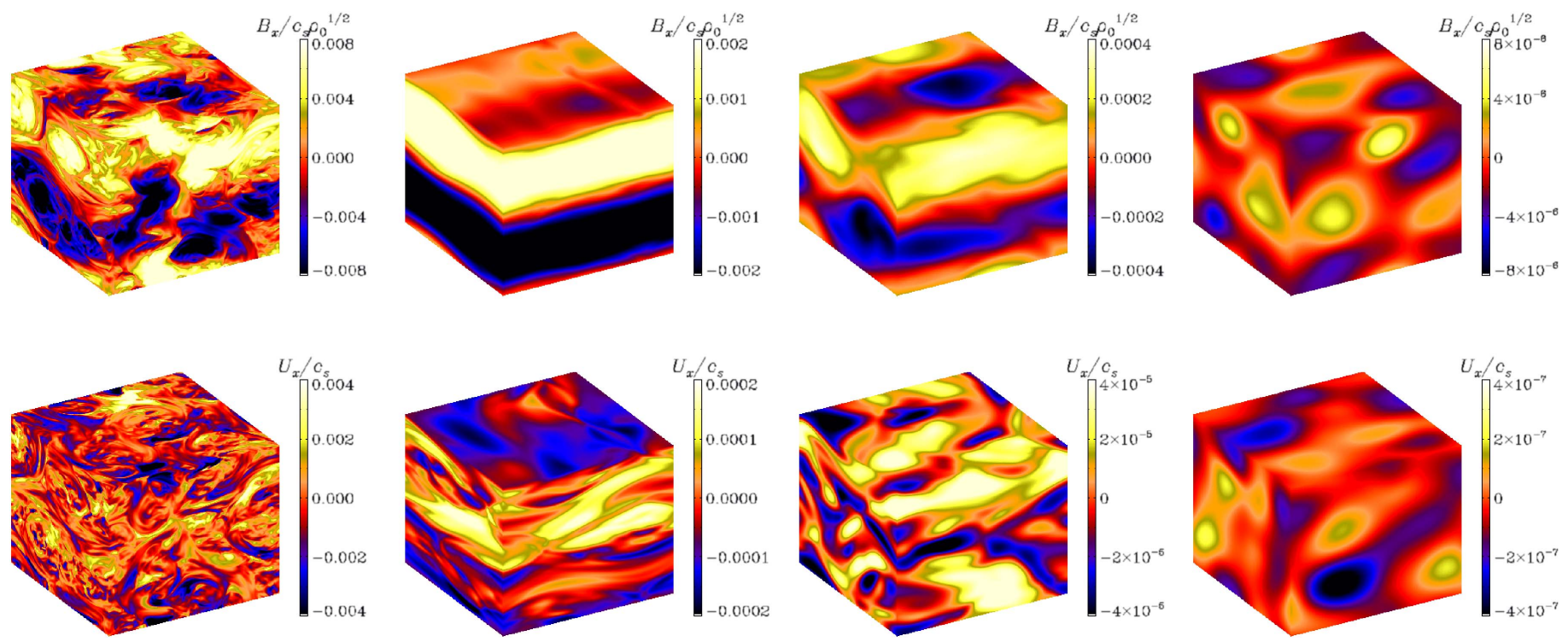

Figure 3. $B_{x}$ and $U_{x}$ on the periphery of the computational domain for (from left to right) $v_{\lambda} / v_{\mu}=700,70,7$, and 0.07 at the last time.

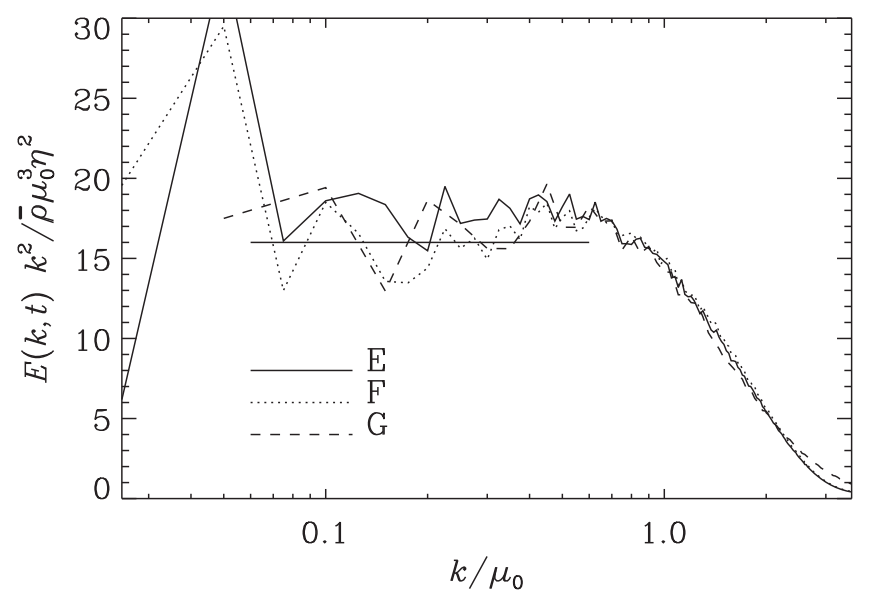

Figure 4. Compensated spectra for Runs E-G.

magnetic fields are similar to cosmic-ray-driven turbulence through the Bell instability (Rogachevskii et al. 2012).

\section{Application to the Early Universe}

Our work has shown that the CME acts as an intermediary to the previously studied cases of decaying hydromagnetic turbulence. It yields the initial conditions: the length scale, $\xi_{\mathrm{M}}=k_{\lambda}^{-1}$, and the field strength, $|\boldsymbol{B}| \approx\left(\mu k_{\lambda} / \lambda\right)^{1 / 2}$; see Equation (7).

We now apply our findings to the case of the cosmological magnetic field. The equations of Section 2 remain unchanged if one uses comoving variables (see Rogachevskii et al. 2017).

The initial value of chiral asymmetry depends on microscopic physics. To establish a model-independent upper limit, it is instructive to write the conservation of total chirality of Equation (6), using the definitions of $\mu$ and $\lambda$ of Section 2, in the form

$$
\left(n_{\mathrm{L}}-n_{\mathrm{R}}\right)+\frac{4 \alpha_{\mathrm{em}}}{\hbar c}\langle\boldsymbol{A} \cdot \boldsymbol{B}\rangle=\text { const. }
$$

Assuming that fermions are in equilibrium with photons, the maximal value of chiral asymmetry in one fermion species is attained when $n_{\mathrm{L}} \approx n_{\gamma}$-the number of photons and $n_{\mathrm{R}} \approx 0$. In this case, the maximal value of magnetic helicity today would be

$$
\left\langle\boldsymbol{B}^{2}\right\rangle \xi_{\mathrm{M}}=\frac{\hbar c}{4 \alpha_{\mathrm{em}}} \frac{g_{0}}{g_{*}} n_{\gamma 0} N_{\mathrm{f}}=5 \times 10^{-38} \frac{N_{\mathrm{f}}}{10} g_{100}^{-1} \mathrm{G}^{2} \mathrm{Mpc}
$$

Here, $g_{0}=3.36$ and $n_{\gamma 0}=2 \zeta(3) / \pi^{2}\left(k_{B} T_{0} / \hbar c\right)^{3}=411 \mathrm{~cm}^{-3}$ is the number of photons today, the factor $N_{\mathrm{f}}$ takes into account that many relativistic fermions with asymmetric populations are present in the plasma, and therefore the total fermion chirality exceeds $n_{\gamma}, g_{100}=g_{*} / 100$ is the effective number of degrees of freedom at temperatures where magnetic fields are generated (in the Standard Model $g_{*}=106.75$ at $T \sim 100 \mathrm{GeV}$ ), and $\zeta(3) \approx 1.202$. If all fermions have comparable asymmetries at high temperature, the estimate (17) is consistent with the lower bound from Dermer et al. (2011). Equations (16) and (17) give an example of fixing the dimensionless factors in Equation (1); the presence of $\alpha_{\mathrm{em}}$ indicates that this is a quantum effect, and the ratio of relativistic degrees of freedom $g_{0} / g_{*}$ appears because $n_{\gamma}$ dilutes as $T^{3}$ while the magnetic helicity decays with scale factor $a$ as $a^{-3}$.

The results of our previous analysis allow us to determine the initial condition for decaying helical turbulence. To this end, we evaluate $v_{\mu}$ and $v_{\lambda}$. The above estimates of the maximal value of the chiral asymmetry give

$$
|\mu| \ll 4 \alpha_{\mathrm{em}} \frac{k_{\mathrm{B}} T}{\hbar c} \approx 1.5 \times 10^{14} T_{100} \mathrm{~cm}^{-1},
$$

where $T_{100}$ is the temperature in units of $100 \mathrm{GeV}$ corresponding to $1.2 \times 10^{15} \mathrm{~K}$. For the magnetic resistivity we use Equation (1.11) of Arnold et al. (2000):

$$
\eta=7.3 \times 10^{-4} \frac{\hbar c^{2}}{k_{\mathrm{B}} T} \approx 4 \times 10^{-9} T_{100}^{-1} \mathrm{~cm}^{2} \mathrm{~s}^{-1} .
$$

Thus, $v_{\mu}=6 \times 10^{5} \mathrm{~cm} \mathrm{~s}^{-1}$, so the number of $e$-folds is $\mathcal{N} \equiv v_{\mu} \mu / H \approx 5 \times 10^{9} g_{100}^{-1 / 2} T_{100}^{-1} \gg 1$, where $H^{-1} \approx 5 \times$ $10^{-11} g_{100}^{-1 / 2} T_{100}^{-2} \mathrm{~s}$ is the Hubble time. 
The chirality flipping rate is a complicated function of temperature below $100 \mathrm{GeV}$, with electromagnetic and weak processes as well as decays of residual Higgs bosons contributing to it; see Appendix D of Boyarsky et al. (2012). However, for a simple numerical estimate at $100 \mathrm{GeV}$, we can extrapolate the rate from the unbroken phase. Using $\Gamma_{\mathrm{f}} / H \approx 800 T_{100}^{-1}$ (Campbell et al. 1992; Joyce \& Shaposhnikov 1997), we find $\Gamma_{\mathrm{f}} / \eta \mu^{2} \approx$ $(800 / \mathcal{N}) T_{100}^{-1} \approx 1.6 \times 10^{-7} g_{100}^{1 / 2} \ll 1$. Although we underestimate the flipping rate in this way, it remains negligible either way.

Next, we determine $\bar{\rho}$ from the Friedmann equation:

$$
\bar{\rho}=\frac{\pi^{2}}{30} g_{*} \frac{\left(k_{\mathrm{B}} T\right)^{4}}{\hbar^{3} c^{5}} \approx 7.6 \times 10^{26} g_{100} T_{100}^{4} \mathrm{~g} \mathrm{~cm}^{-3},
$$

and we find

$$
\lambda=3 \hbar c\left(\frac{8 \alpha_{\mathrm{em}}}{k_{\mathrm{B}} T}\right)^{2} \approx 1.3 \times 10^{-17} T_{100}^{-2} \mathrm{~cm} \mathrm{erg}^{-1} .
$$

As a result, $v_{\lambda} \approx 1.5 \times 10^{9} \mathrm{~cm} \mathrm{~s}^{-1} \gg v_{\mu}$ and $v_{\lambda} \ll c_{\mathrm{s}} \approx$ $2 \times 10^{10} \mathrm{~cm} \mathrm{~s}^{-1}$, so we are in regime I where turbulence develops. Finally, we estimate the length of the inertial range of chiral magnetically driven turbulence from

$$
v_{\mu} / v_{\lambda}=\eta(\bar{\rho} \lambda)^{1 / 2} \approx g_{100}^{1 / 2} / 2400 .
$$

Equation (13) with $\sqrt{C_{\mu} / C_{\lambda}} \approx 4$ gives $\mu / k_{\lambda} \approx 600 g_{100}^{-1 / 2}$. So we expect a $k^{-2}$ spectrum covering almost three orders of magnitude.

\section{Conclusions}

We have shown that in chiral magnetohydrodynamics, magnetic field evolution proceeds in distinct stages: (i) smallscale chiral dynamo instability; (ii) first nonlinear stage when the Lorentz force drives small-scale turbulence; (iii) development of inverse energy transfer with a $k^{-2}$ magnetic energy spectrum over the range $k_{\lambda}<k<\mu$; and (iv) generation of large-scale magnetic field by chiral magnetically driven turbulence, decrease of the chemical potential, saturation, and eventual decay. This process acts as an intermediary to decaying hydromagnetic turbulence.

The application to the early universe results in a limit on magnetic helicity, depending on the plasma particle content at high temperatures. Larger values of $\left\langle\boldsymbol{B}^{2}\right\rangle \xi_{\mathrm{M}}$ can only be envisaged if $\left\langle\boldsymbol{B}^{2}\right\rangle$ and $\xi_{\mathrm{M}}$ are constrained separately, e.g., as a fraction of the radiation energy of the universe and a fraction of the Hubble horizon (Kahniashvili et al. 2013).

We thank Tanmay Vachaspati for useful discussions. Support through the NSF Astrophysics and Astronomy Grant Program (grant Nos. 1615100 and 1615940), the Research Council of Norway (FRINATEK grant No. 231444), the Georgian NSF FR/264/6-350/14, and the European Research Council (grant No. 694896) are gratefully acknowledged. We acknowledge the allocation of computing resources provided by the Swedish National Allocations Committee at the Center for Parallel Computers at the Royal Institute of Technology in Stockholm. This work utilized the Janus supercomputer, which is supported by the National Science Foundation (award No. CNS-0821794), the University of Colorado Boulder, the University of Colorado Denver, and the National Center for Atmospheric Research. The Janus supercomputer is operated by the University of Colorado Boulder.

\section{ORCID iDs}

Axel Brandenburg (iD https://orcid.org/0000-0002-7304-021X Jennifer Schober (D) https://orcid.org/0000-0001-7888-6671 Igor Rogachevskii (i) https://orcid.org/0000-0001-7308-4768 Tina Kahniashvili (iD https://orcid.org/0000-0003-0217-9852 Oleg Ruchayskiy (iD https://orcid.org/0000-0001-8073-3068 Nathan Kleeorin (i) https://orcid.org/0000-0002-5744-1160

\section{References}

Aharonian, F., Akhperjanian, A. G., Bazer-Bachi, A. R., et al. 2006, Natur, 440, 1018

Alekseev, A. Y., Cheianov, V. V., \& Fröhlich, J. 1998, PhRvL, 81, 3503

Arnold, P., Moore, G. D., \& Yaffe, L. G. 2000, JHEP, 11, 001

Banerjee, R., \& Jedamzik, K. 2004, PhRvD, 70, 123003

Biskamp, D., \& Müller, W.-C. 1999, PhRvL, 83, 2195

Boyarsky, A., Fröhlich, J., \& Ruchayskiy, O. 2012, PhRvL, 108, 031301

Boyarsky, A., Fröhlich, J., \& Ruchayskiy, O. 2015, PhRvD, 92, 043004

Brandenburg, A. 2001, ApJ, 550, 824

Brandenburg, A. 2010, MNRAS, 401, 347

Brandenburg, A., Enqvist, K., \& Olesen, P. 1996, PhRvD, 54, 1291

Brandenburg, A., \& Kahniashvili, T. 2017, PhRvL, 118, 055102

Brandenburg, A., Kahniashvili, T., \& Tevzadze, A. G. 2015, PhRvL, 114, 075001

Campbell, B. A., Davidson, S., Ellis, J., \& Olive, K. A. 1992, PhLB, 297, 118

Christensson, M., Hindmarsh, M., \& Brandenburg, A. 2001, PhRvE, 64, 056405

Dermer, C. D., Cavadini, M., Razzaque, S., Finke, J. D., Chiang, J., \& Lott, B. 2011, ApJL, 733, L21

Durrer, R., \& Caprini, C. 2003, JCAP, 0311, 010

Durrer, R., \& Neronov, A. 2013, A\&ARv, 21, 62

Dvornikov, M., \& Semikoz, V. B. 2017, PhRvD, 95, 043538

Fröhlich, J., \& Pedrini, B. 2000, in Int. Conf. on Mathematical Physics, ed. A. S. Fokas et al. (Singapore: World Scientific)

Fukushima, K., Kharzeev, D. E., \& Warringa, H. J. 2008, PhRvD, 78, 074033

Galtier, S., Nazarenko, S. V., Newell, A. C., \& Pouquet, A. 2000, JP1Ph, 63,447

Giovannini, M. 2013, PhRvD, 88, 063536

Hirono, Y., Kharzeev, D., \& Yin, Y. 2015, PhRvD, 92, 125031

Joyce, M., \& Shaposhnikov, M. 1997, PhRvL, 79, 1193

Kahniashvili, T., Tevzadze, A. G., Brandenburg, A., \& Neronov, A. 2013, PhRvD, 87, 083007

Kharzeev, D. E. 2014, PrPNP, 75, 133

Krause, F., \& Rädler, K.-H. 1980, Mean-field Magnetohydrodynamics and Dynamo Theory (Oxford: Pergamon)

Miransky, V. A., \& Shovkovy, I. A. 2015, PhRv, 576, 1

Moffatt, H. K. 1978, Magnetic Field Generation in Electrically Conducting Fluids (Cambridge: Cambridge Univ. Press)

Pavlović, P., Leite, N., \& Sigl, G. 2017, PhRvD, 96, 023504

Redlich, A. N., \& Wijewardhana, L. C. R. 1985, PhRvL, 54, 970

Rogachevskii, I., Kleeorin, N., Brandenburg, A., \& Eichler, D. 2012, ApJ, 753, 6

Rogachevskii, I., Ruchayskiy, O., Boyarsky, A., et al. 2017, ApJ, submitted (arXiv:1705.00378)

Schober, J., Rogachevskii, I., Brandenburg, A., et al. 2017, ApJ, submitted

Tashiro, H., Chen, W., Ferrer, F., \& Vachaspati, T. 2014, MNRAS, 445, L41

Tashiro, H., \& Vachaspati, T. 2015, MNRAS, 448, 299

Tashiro, H., Vachaspati, T., \& Vilenkin, A. 2012, PhRvD, 86, 105033

Taylor, A. M., Vovk, I., \& Neronov, A. 2011, A\&A, 529, A144

Vilenkin, A. 1980, PhRvD, 22, 3080 